\title{
Benefit of Postoperative Radiotherapy for Patients With Nonmetastatic Adrenocortical Carcinoma: A Population-Based Analysis
}

\author{
Kan Wu, MD ${ }^{1, *} ;$ Xu Liu, $\mathrm{PhD}^{2, *}$; Zhihong Liu, MD ${ }^{1}$; Yiping Lu, MD ${ }^{1}$; Xianding Wang, MD'; \\ and Xiang Li, MD ${ }^{1}$
}

\begin{abstract}
Background: Adrenocortical carcinoma (ACC) is an aggressive cancer with high recurrence rates and poor prognosis, even after radical surgery. The survival benefit of adjuvant radiotherapy $(R T)$ in patients with ACC has not been well explored. The aim of this study was to evaluate the effect of adjuvant RT on the survival outcome of patients with ACC. Patients and Methods: All patients with nonmetastatic ACC who underwent complete resection were identified from the SEER database (2004-2016). Overall survival (OS) was estimated using the Kaplan-Meier method. Multivariable Cox regression analysis was performed to identify prognostic factors associated with survival. Results: Of 365 patients with nonmetastatic ACC, 55 (15.1\%) received adjuvant RT and the remainder underwent surgery alone. Patient characteristics were similar between the 2 groups, but those with a higher disease stage were more likely to receive adjuvant RT. Overall, patients receiving RT seemed to have better survival compared with the non-RT group (3-year OS rate, $63.1 \%$ vs $52.8 \%$; $P<$.062). After adjustment for confounding factors, adjuvant RT was indeed associated with a $48 \%$ decreased risk of death (hazard ratio, $0.52 ; 95 \% \mathrm{Cl}, 0.29-0.91 ; P=.023$ ) for all patients. In addition, adjuvant RT may confer a survival benefit only in patients with a high risk of recurrence (3-year OS rate, $55.1 \%$ vs $40.0 \% ; P=.048$ ) rather than in those with low/moderate-risk ACC $(P=.559)$. Conclusions: Our findings suggest that adjuvant RT may be associated with improved survival in patients with nonmetastatic ACC who underwent radical surgery, especially those with high risk of recurrence.
\end{abstract}

J Natl Compr Canc Netw 2021;19(12):1425-1432 doi: $10.6004 /$ jnccn.2021.7035

\footnotetext{
${ }^{1}$ Department of Urology, Institute of Urology, and ${ }^{2}$ Laboratory of Molecular Diagnosis of Cancer, Clinical Research Center for Breast, West China Hospital, Sichuan University, Chengdu, China.
}

*These authors contributed equally to this work.

\section{Background}

Adrenocortical carcinoma (ACC) is a rare but highly aggressive tumor with an estimated incidence of 0.7 to 2.0 per 1,000,000 adult individuals annually. ${ }^{1}$ Radical surgery is still the mainstay treatment of patients with nonmetastatic ACC. ${ }^{2}$ However, approximately two-thirds of patients with ACC may experience locoregional recurrence after complete surgical resection. ${ }^{3}$ Even after R0 resection with negative margins, approximately $30 \%$ of patients may still experience a tumor recurrence, leading to a poor prognosis. ${ }^{4}$ Therefore, there is an urgent need for adjuvant treatment intensification to prevent disease recurrence and improve survival outcomes.

Currently, mitotane monotherapy, cytotoxic drugs, and radiotherapy (RT) are used in the adjuvant setting. ${ }^{5}$ However, there is a lack of completely convincing evidence to show whether adjuvant mitotane and chemotherapy will decrease local recurrence and improve survival. ${ }^{5}$ Furthermore, aggressive medical therapy often comes with diverse adverse effects and heavy economic burdens. Because of the limited treatment alternatives, adjuvant RT after operative resection has been considered as a potential option for reducing tumor recurrence and prolonging survival in patients with nonmetastatic ACC.

Because of the rarity of this disease, the role of adjuvant RT after surgery for nonmetastatic ACC has not been well explored, Historically, ACC has been considered a relatively radiation-resistant cancer based on the disappointing results of previous case series. ${ }^{6}$ However, utilizing modern radiation techniques, more retrospective studies with small sample sizes have hinted that adjuvant RT may prevent local failures in selected patients with a high risk of recurrence. ${ }^{7-10}$ A large US hospital-based investigation further indicated that adjuvant RT may improve the survival outcome of patients with nonmetastatic ACC and positive surgical margins, but only $14.4 \%$ of patients currently receive adjuvant RT after complete resection. ${ }^{11}$ Recently, a retrospective propensity-matched analysis demonstrated that adjuvant RT could provide significant improvements in local recurrence-free and overall survival (OS) regardless of margin status. ${ }^{12}$ However, the analysis 
failed to determine which patient subgroups might really benefit from adjuvant RT, and the small numbers of patients limited the generalization.

Given this background, we intended to evaluate the effect of adjuvant RT after radical surgery on patients with nonmetastatic ACC using a large population-based database. In addition, we sought to identify a subset of patients with ACC who may be more likely to benefit from adjuvant radiation based on their tumor characteristics.

\section{Patients and Methods}

\section{Study Population}

The NCI's SEER database is a nationwide cancer registry that provides cancer-specific information for approximately $28 \%$ of the US population. We identified patients diagnosed with nonmetastatic ACC from January 1, 2004, to December 31, 2016.

Inclusion criteria included the following: (1) primary site C74.0 with histology codes 8010,8140 , and 8370 , or C74.9 with histology code 8370; (2) localized or regional disease at initial diagnosis (stage I, II, or III); (3) unilateral ACC confirmed by histology that was removed via radical adrenalectomy (labeled with codes 40 [total surgical removal of primary site] and 60 [radical surgery]); and (4) adult at diagnosis (aged $\geq 18$ years), with ACC labeled as the first and only primary tumor. Patients with unknown information on adjuvant RT, those who died early after surgery $(<30$ days), and those with unknown survival data were excluded from this cohort.

\section{Clinical Variables}

Variables included patient age, sex, race, marital status, tumor laterality, year of diagnosis, tumor size, tumor stage, lymph node status, tumor grade, adjuvant therapy (RT or chemotherapy), survival time, and survival status. The tumor stages were reevaluated based on the European Network for the Study of Adrenal Tumors (ENSAT) staging system. ENSAT I and II were combined and treated as the reference. Age at diagnosis was divided into 2 groups: $\leq 55$ years and $>55$ years. Tumor size was classified as $<10.0$ and $\geq 10.0 \mathrm{~cm}$, whereas tumors $>30.0 \mathrm{~cm}$ were treated as the coding errors. Lymph node status was categorized into node-positive, node-negative, and unknown status. Tumor grade was categorized into well-differentiated I/II, poor to undifferentiated III/IV, and unknown. Adjuvant RT was categorized as yes or no, and adjuvant chemotherapy was categorized as yes or no/unknown. The primary outcome was OS for patients with ACC after radical surgery.

\section{Statistical Analysis}

Patients were grouped based on treatment type: surgery alone versus surgery plus adjuvant RT. Patient characteristics were described as count (percentage) for categorical variables or median (range) for continuous variables. The distributions between the 2 groups were compared using the Mann-Whitney $U$ test, chi-square analysis, and Fisher exact tests, as appropriate. OS was estimated using the KaplanMeier method and compared using the log-rank test. A univariable and multivariable Cox regression analysis was applied to examine the independent effects of adjuvant RT and other covariates on the survival outcomes and expressed as a hazard ratio (HR) with its $95 \%$ confidence interval.

\section{Exploratory Analyses}

Regarding the high-risk recurrence features of patients with localized ACC, the NCCN Clinical Practice Guidelines in Oncology (NCCN Guidelines) for Neuroendocrine and Adrenal Tumors (Version 2.2020) include positive margins, Ki-67 $>10 \%$, rupture of a capsule, large size, and high grade, ${ }^{13}$ and the European Society of Endocrinology Clinical Practice Guidelines include stage III, $\mathrm{R} 1$ resection, or Ki-67>10\%. ${ }^{5}$ Based on the risk factors available in the SEER database, as well as the fact that age $>55$ years and node-positive status are known to decrease survival, the following 5 variables were considered as high-risk characteristics for recurrence or death: ENSAT stage III, large tumor size $(\geq 10 \mathrm{~cm})$, node-positive status, high-grade histology, and/or age $>55$ years. All patients were then divided into 2 prognostic groups: the low/moderate-risk group (risk factor $\leq 1$ ) and the high-risk group (risk factors $\geq 2$ ). Finally, to identify the patients most likely to benefit from adjuvant RT, we planned to performed an exploratory subgroup analysis to investigate the benefits for each risk group. We used SPSS Statistics, version 22.0 (IBM Corp.) and $\mathrm{R}$ version 3.5.1 (R Foundation for Statistical Computing) to perform all statistical tests; $P<.05$ was considered statistically significant.

\section{Results}

\section{Patient Characteristics}

A total of 365 patients with nonmetastatic ACC who underwent radical adrenalectomy were identified based on the inclusion and exclusion criteria. Of these, 55 (15.1\%) received postoperative adjuvant radiation and $310(84.9 \%)$ underwent surgical resection alone. Median age at initial diagnosis was 54.5 years (range, 18-89 years) for the general cohort. Median tumor size was $10.5 \mathrm{~cm}$ (range, 1.2-30.0 cm). Most patients were female $(62.2 \%$ ) and White $(82.2 \%)$. According to the final pathology, $14.8 \%$ of patients harbored high-grade histology, and positive lymph node status was identified in $6.3 \%$ of patients. 
The baseline clinicopathologic characteristics of patients by treatment group are presented in Table 1. No significant differences between the 2 groups were observed for age at diagnosis, sex, race, marital status, tumor size, tumor grade, or lymph node status. Patients with higher-stage disease $(54.5 \%$ vs $39.0 \% ; P=.031)$ and those treated with chemotherapy $(67.3 \%$ vs $33.9 \%$; $P<.001)$ were more likely to receive adjuvant RT compared with patients with lower-stage disease and those not treated with chemotherapy, respectively. In addition adjuvant RT was administered more in patients with right-sided ACC.

\section{Survival Outcomes}

The SEER database does not record information regarding disease recurrence. Of the 365 patients, 175 (47.9\%) died during the follow-up period (median time, 26 months). The 1-, 3-, and 5-year OS rates for patients who

\section{Table 1. Patient Characteristics}

\begin{tabular}{|c|c|c|c|}
\hline Variable & $\begin{array}{c}\text { Surgery Alone } \\
n(\%)\end{array}$ & $\begin{array}{c}\text { Surgery + Radiation } \\
\mathrm{n}(\%)\end{array}$ & $P$ Value \\
\hline Total, $n$ & 310 & 55 & \\
\hline Age, median (range) & $54.5(18.0-89.0)$ & $53.0(18-85)$ & .445 \\
\hline$\leq 55 y$ & $160(51.6)$ & $32(58.2)$ & .369 \\
\hline$>55 y$ & $150(48.4)$ & $23(41.8)$ & \\
\hline Sex & & & .116 \\
\hline Female & $198(63.9)$ & $29(52.7)$ & \\
\hline Male & $112(36.1)$ & $26(47.3)$ & \\
\hline Race & & & .399 \\
\hline White & $257(82.9)$ & $43(78.2)$ & \\
\hline Other/Unknown & $53(17.1)$ & $12(21.8)$ & \\
\hline Marital status & & & .914 \\
\hline Married & $194(62.6)$ & $34(61.8)$ & \\
\hline Single/Unknown & $116(37.4)$ & $21(38.2)$ & \\
\hline Laterality & & & .008 \\
\hline Left & $173(55.8)$ & $20(36.4)$ & \\
\hline Right & $137(44.2)$ & $35(63.6)$ & \\
\hline Tumor size, median (range) & $10.5(1.8-30.0)$ & $10.3(1.2-22.5)$ & .219 \\
\hline$<10 \mathrm{~cm}$ & $127(41.0)$ & $25(45.5)$ & .752 \\
\hline$\geq 10 \mathrm{~cm}$ & $169(54.5)$ & $27(49.1)$ & \\
\hline Unknown & $14(4.5)$ & $3(5.4)$ & \\
\hline Disease stage & & & .031 \\
\hline $1 / I I$ & $189(61.0)$ & $25(45.5)$ & \\
\hline III & $121(39.0)$ & $30(54.5)$ & \\
\hline Tumor grade & & & .167 \\
\hline $1 / 2$, well-differentiated & $18(5.8)$ & $7(12.7)$ & \\
\hline $3 / 4$, poor to undifferentiated & $47(15.2)$ & $7(12.7)$ & \\
\hline Unknown & $245(79.0)$ & $41(74.6)$ & \\
\hline Lymph node status & & & .772 \\
\hline Negative & $57(18.4)$ & $12(21.8)$ & \\
\hline Positive & $19(6.1)$ & $4(7.3)$ & \\
\hline Unknown & $234(75.5)$ & $39(70.9)$ & \\
\hline Chemotherapy & & & $<.001$ \\
\hline No/Unknown & $205(66.1)$ & $18(32.7)$ & \\
\hline Yes & 105 (33.9) & $37(67.3)$ & \\
\hline
\end{tabular}

Abbreviation: ACC, adrenocortical carcinoma. 
had surgery alone were $80.4 \%, 52.8 \%$, and $43.6 \%$ compared with $90.5 \%, 63.1 \%$, and $55.7 \%$, respectively, for those who received adjuvant RT after surgery (log-rank $P<.062$; Figure 1). After adjusting for confounding factors, adjuvant RT was significantly associated with improved survival (HR, 0.52; 95\% CI, 0.29-0.91; $P=.023$ ) (Figure 1, Table 2).

As shown in Figure 2, the effect of adjuvant RT on survival outcomes in all prespecified subgroups was then examined. After surgical resection, adjuvant RT provided a significant survival difference at 3 years for women $(64.7 \%$ vs $53.5 \%$; HR, 0.38; 95\% CI, $0.16-0.95 ; P=.037)$ and patients with small tumor size (74.2\% vs $59.0 \%$; HR, 0.30 ; 95\% CI, $0.09-0.96 ; P=.042$ ), those with a higher stage of disease $(57.3 \%$ vs $36.4 \%$; HR, $0.42 ; 95 \% \mathrm{CI}, 0.21-0.84 ; P=.015)$, and those treated using chemotherapy (59.6\% vs $45.2 \%$; HR, 0.45 ; 95\% CI, 0.23-0.88; $P=.020$ ).

Table 2 shows the results of the univariable and multivariable Cox regression model for OS in patients with nonmetastatic ACC who underwent complete surgical resection. In univariable analysis, age $>55$ years, male sex, large tumor size ( $\geq 10 \mathrm{~cm}$ ), stage III disease, and positive lymph node status were associated with decreased survival (all $P<.05$ ). Meanwhile, adjuvant RT, race, married status, tumor laterality, tumor grade, and receipt of chemotherapy were not significantly associated with survival outcomes (all $P>.05$ ). In the multivariable Cox analysis, after adjustment for confounding factors, age $>55$ years (HR, 1.53; 95\% CI, 1.11-2.12; $P=.010$ ), high disease stage (HR, 1.61; 95\% CI, 1.14-2.27; $P=.007$ ), nodal metastases (HR, 2.69; 95\% CI, 1.45-4.99; $P=.002$ ), and receipt of chemotherapy (HR, 1.42; 95\% CI, 1.02-1.99; $P=.041$ ) were independently associated with worse survival. However, after adjusting for other tumor characteristics, adjuvant RT was associated with a statistically significant $48 \%$

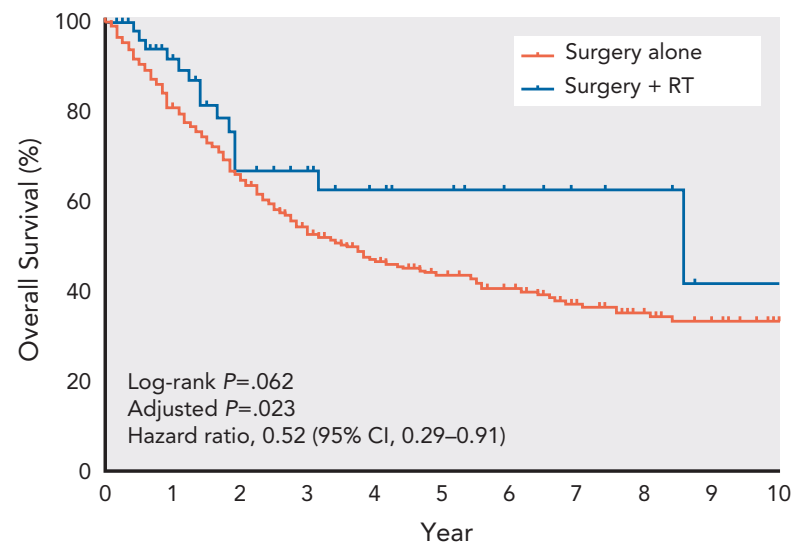

Figure 1. Overall survival in all patients treated with surgery alone versus surgery plus RT.

Abbreviation: $\mathrm{RT}$, radiotherapy. decreased risk of death (HR, 0.52; 95\% CI, 0.29-0.91; $P=.023)$ for all patients with nonmetastatic ACC who underwent radical surgery.

\section{Exploratory Analyses}

Based on the known high-risk characteristics of recurrence or death (stage III disease, large tumor size, nodepositive status, high-grade histology, and age $>55$ years), all patients were classified into 2 prognostic groups: those with $\leq 1$ risk factor were sorted into the low/moderate-risk group ( $\mathrm{n}=177$ ), and patients with $\geq 2$ risk factors were sorted into the high-risk group $(\mathrm{n}=188)$. The 2 risk subgroups had a distinct difference in 3-year OS rate: $67.4 \%$ in the low/moderate-risk group versus $42.8 \%$ in the high-risk group, respectively $(P<.001$; Figure $3 \mathrm{~A})$. In the subgroup analysis (Figure $3 \mathrm{~B}, \mathrm{C}$ ), adjuvant RT was significantly associated with prolonged survival in the high-risk group (3-year OS rate: $55.1 \%$ vs $40.0 \%$; $P=.048$ ). However, adjuvant RT was not correlated with survival outcomes in the low/moderate-risk group $(P=.559)$.

\section{Discussion}

Using a large administrative database, we found that adjuvant RT after radical resection could confer a survival advantage in patients with nonmetastatic ACC, especially those with a high risk of recurrence. Furthermore, our study indicated that the survival benefit of adjuvant RT for ACC should be discussed based on individualized patient risk factors. Our findings support the view that adjuvant radiation seems to be particularly justified in selected patients who are at perceived high risk for recurrence.

Evaluating the role of adjuvant RT in nonmetastatic ACC after surgery has been challenging. Early series exploring the potential benefit of adjuvant radiation in terms of local recurrence control drew conflicting conclusions with null results, ${ }^{14-17}$ leading to ACC being treated as a radiation-resistant tumor, and therefore a very small proportion of patients were treated using adjuvant radiation. However, several institutions utilizing modern radiation technology have shown that adjuvant radiation in ACC after surgery can reduce local recurrence and should be used in the adjuvant setting. ${ }^{7-10}$ A recent meta-analysis focused on adjuvant RT after surgical resection for ACC and provided pooled data from these retrospective studies. ${ }^{18}$ The meta-analysis found that adjuvant radiation could be safely administered, with low adverse effects, and could significantly decrease local recurrence, but it did not significantly affect the distant metastases and OS. ${ }^{18}$ Recently, a single-institution study even found that adjuvant radiotherapy could not only improve local control, but also improve the OS of patients with ACC. ${ }^{12}$ However, these studies have had limited quality assurance or have been hampered by small ample sizes. Given the rarity of the disease, completing a clinical trial with 


\begin{tabular}{|c|c|c|c|c|c|}
\hline Variable & 3-Year OS, \% & $\mathrm{HR}^{\mathrm{a}}(95 \% \mathrm{Cl})$ & $P$ Value & $\mathrm{HR}^{\mathrm{a}}(95 \% \mathrm{Cl})$ & $P$ Value \\
\hline \multicolumn{6}{|l|}{ Treatment } \\
\hline Surgery alone & 52.8 & Ref & & Ref & \\
\hline Surgery + radiation & 63.1 & $0.61(0.36-1.03)$ & .066 & $0.52(0.29-0.91)$ & .023 \\
\hline$\leq 55 y$ & 59.3 & Ref & & Ref & \\
\hline$>55 y$ & 49.2 & $1.46(1.08-1.96)$ & .013 & $1.53(1.11-2.12)$ & .010 \\
\hline \multicolumn{6}{|l|}{ Sex } \\
\hline Female & 56.0 & Ref & & Ref & \\
\hline Male & 52.2 & $1.39(1.03-1.88)$ & .030 & $1.35(0.98-1.86)$ & .071 \\
\hline \multicolumn{6}{|l|}{ Marital status } \\
\hline Married & 52.2 & Ref & & - & \\
\hline Single & 58.3 & $0.78(0.57-1.07)$ & .117 & - & - \\
\hline \multicolumn{6}{|l|}{ Laterality } \\
\hline Left & 50.5 & Ref & & - & \\
\hline Right & 58.4 & $0.97(0.72-1.31)$ & .854 & - & - \\
\hline \multicolumn{6}{|l|}{ Tumor size } \\
\hline$<10 \mathrm{~cm}$ & 63.1 & Ref & & Ref & \\
\hline$\geq 10 \mathrm{~cm}$ & 49.8 & $1.51(1.10-2.08)$ & .012 & $1.34(0.95-1.88)$ & .094 \\
\hline Unknown & 55.5 & $2.03(0.95-4.35)$ & .067 & $1.88(0.81-4.32)$ & .140 \\
\hline \multicolumn{6}{|l|}{ Lymph node status } \\
\hline Negative & 52.2 & Ref & & Ref & \\
\hline Positive & 13.1 & $3.80(2.12-6.81)$ & $<.001$ & $2.69(1.45-4.99)$ & .002 \\
\hline Unknown & 58.2 & $0.98(0.67-1.45)$ & .933 & $1.10(0.72-1.66)$ & .666 \\
\hline \multicolumn{6}{|l|}{ Chemotherapy } \\
\hline No/Unknown & 57.2 & Ref & & Ref & \\
\hline Yes & 49.7 & $1.33(0.98-1.80)$ & .065 & $1.42(1.02-1.99)$ & .041 \\
\hline
\end{tabular}

Variables with $P \leq .10$ in univariable analysis were included in the multivariable Cox regression.

Abbreviations: ACC, adrenocortical carcinoma; HR, hazard ratio; OS, overall survival.

${ }^{a} \mathrm{HR}>1.0$ denotes higher mortality risk.

adequate accrual may be difficult. Therefore, large-scale, real-world studies may be of value for assessing the effect of adjuvant radiation on ACC and could provide important guidance for treatment decisions.

Unfortunately, a prior analysis of the SEER database did not find any association between RT and improved overall or cancer-specific survival in patients with ACC; it failed to clarify the value of RT in the adjuvant setting because it simply assessed the effect of RT on all patients with ACC, including nonsurgical patients and those with metastatic ACC. ${ }^{19}$ Furthermore, a large patient cohort analysis using the National Cancer Database showed that 

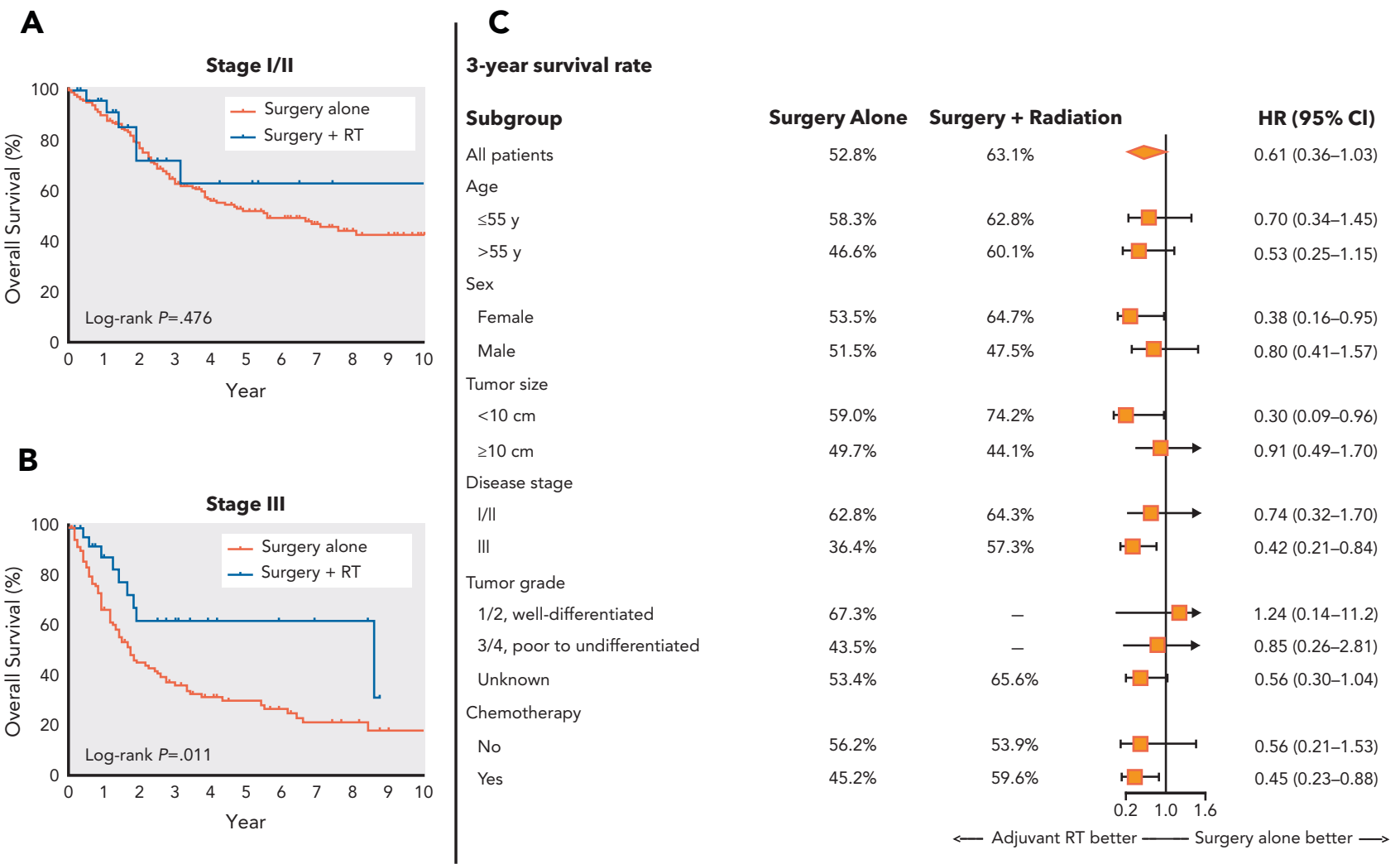

Figure 2. Effect of adjuvant RT on survival outcomes in patients with (A) stage I/II and (B) stage III adrenocortical carcinoma, and (C) all prespecified subgroups.

Abbreviations: $H R$, hazard ratio; $R T$, radiotherapy.

only $14.4 \%$ of patients with nonmetastatic ACC underwent surgical resection followed by adjuvant RT, whereas the remaining patients received surgery alone. ${ }^{11}$ This observation was in accordance with our finding that postoperative adjuvant radiation (14.9\%) was not routinely used as a treatment option. In addition, the analysis found that compared with surgery alone, adjuvant radiation was associated with a $40 \%$ decreased risk of death in patients with positive surgical margins, highlighting the potential value of RT in the adjuvant setting. ${ }^{11}$ However, the analysis failed to consider and combine more high-risk characteristics for recurrence, including stage III disease, tumor grade, and large tumor size, which limited the scope of the findings. We hypothesized that patients with a higher risk of local recurrence may be more likely to benefit from adjuvant RT.

In this study, we found that adjuvant RT may only confer a survival benefit in patients at higher risk of recurrence rather than in those with low/moderate-risk ACC because the potential residual tumor foci after surgery may be eradicated after RT. Our findings confirm the assumption that improvements in local control with adjuvant radiation can translate into benefits in survival outcomes for patients with ACC, which have been shown in other cancer types. ${ }^{20-22}$ Furthermore, our results are also supported by current recommendations ${ }^{5,13}$ in that they encourage adjuvant radiation for patients with highrisk ACC but oppose the routine use of adjuvant radiation in patients with a low/moderate risk of recurrence. Although combinations of risk factors could be generated to define the subgroup classification, there is no convincing evidence to support these patient selection criteria in treatment decision-making. Future studies should focus on identifying suitable candidates who may benefit more from adjuvant RT, according to the clinicopathologic or molecular features that will predict response to RT.

There are several limitations in this study. First, although this study is based on the SEER database, which covers approximately $28 \%$ of the American population, adjuvant RT is infrequently administered in patients with nonmetastatic ACC who have undergone complete resection, ${ }^{19}$ resulting in a relatively small RT group. Second, information on the type of surgical procedure, patient performance status, and comorbidities are not available in the SEER database. In addition, several prognostic factors were not recorded, such as the hormonal functional status, ${ }^{23} \mathrm{Ki}-67$ index, ${ }^{24}$ resection status, ${ }^{25}$ and a detailed scheme of adjuvant radiation or 
A

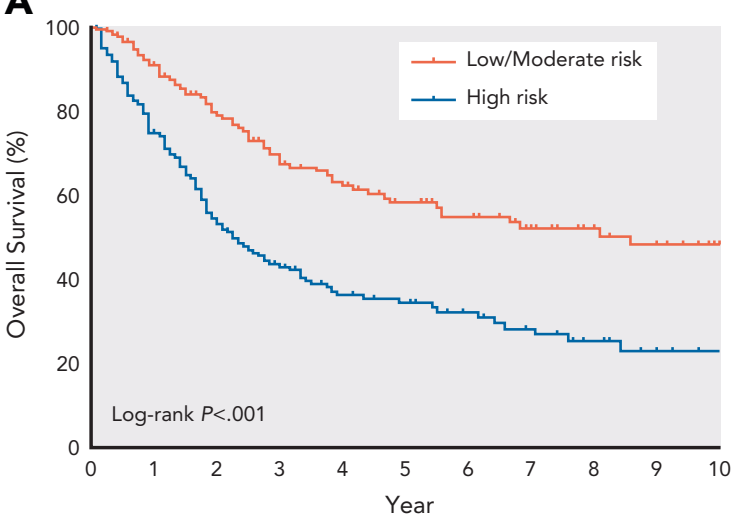

B
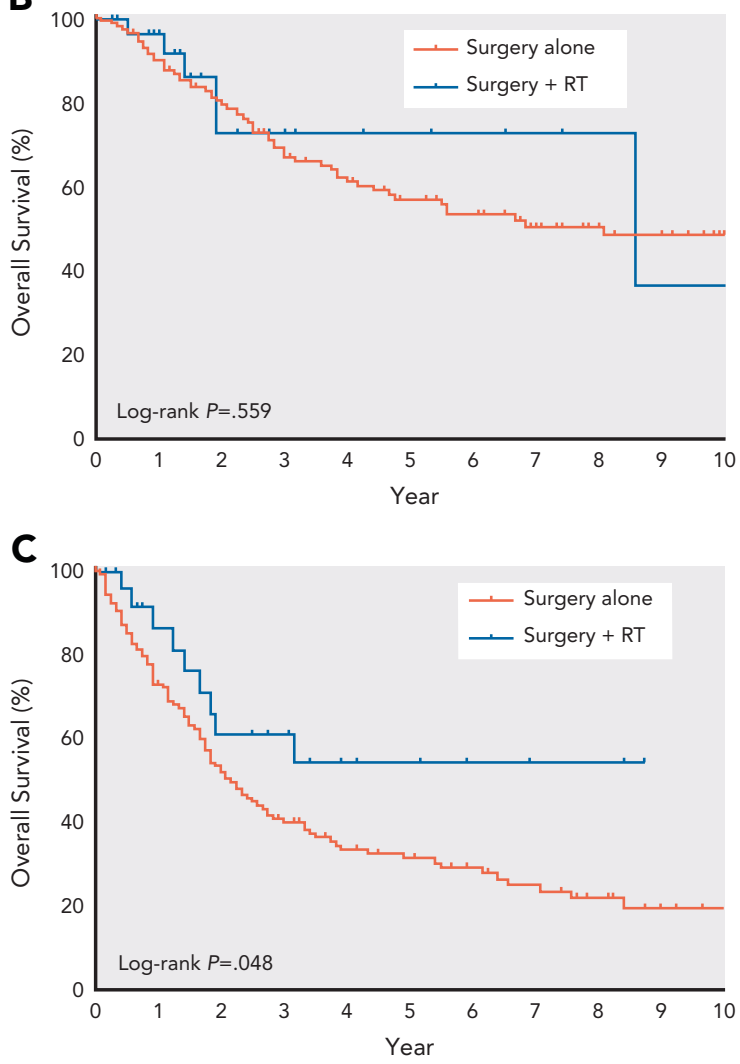

Figure 3. Comparison of overall survival in (A) all patients and those with (B) low/moderate and (C) high risk of recurrence. Abbreviation: RT, radiotherapy.

other systemic therapy; these factors may contribute to patient prognosis. Most important, we failed to examine the effect of adjuvant radiation on local recurrence-free and disease-free survival outcomes in patients with nonmetastatic ACC after radical surgery in this study, because the SEER database lacks information regarding disease recurrence and metastasis. However, we believe that OS is an important and powerful oncologic outcome. It is worth noting that the survival benefit of RT may be mitigated because more patients in the RT group received chemotherapy $(67 \%)$ compared with the surgery group (34\%). According to guidelines from $\mathrm{NCCN}^{13}$ and the European Society of Endocrinology, ${ }^{5}$ one might safely assume that chemotherapy was administered after disease recurrence or metastasis, because chemotherapy was associated with worse survival in this cohort. This finding further highlights the importance of locoregional control.

\section{Conclusions}

Overall, we found that adjuvant RT after radical surgery may be associated with improved survival outcomes in patients with nonmetastatic ACC, especially in those with a high risk of recurrence. Therefore, our findings suggest that adjuvant RT could be routinely used in selected patients with high-risk ACC. In view of the findings of this retrospective study, future multicenter, prospective clinical investigations are urgently needed to clarify the value of adjuvant RT in ACC.

\section{Acknowledgments}

The authors appreciate the SEER database for providing highquality clinical data for our research.

Submitted November 25, 2020; final revision received February 28, 2021; accepted for publication March 3, 2021

Author contributions: Study concept and design: Li, Wang. Visualization: Wu, X. Liu, Z. Liu. Funding acquisition: Li. Data acquisition: Wu, X. Liu. Data analysis and interpretation: Wu, X. Liu. Software: Wu, X. Liu. Supervision: Wang, Li. Project administration: Li. Manuscript preparation: Wu, X. Liu. Critical revision: Lu, Wang, Li.

Disclosures: The authors have disclosed that they have not received any financial consideration from any person or organization to support the preparation, analysis, results, or discussion of this article.

Funding: This work was supported by the 1.3.5 Project for Disciplines of Excellence, West China Hospital, Sichuan University (grant numbers 2018HXFH049, 2019-075, ZY2016104, and 2021HXFH007).

Correspondence: Xiang Li, MD, Department of Urology, Institute of Urology, West China Hospital, Sichuan University, 37 Guoxue Lane, Chengdu, Sichuan 610041, China. Email: xiangli.87@163.com; and Xianding Wang, MD, Department of Urology, Institute of Urology, West China Hospital, Sichuan University, 37 Guoxue Lane, Chengdu, Sichuan 610041, China. Email: xiandingwang@outlook.com

\section{References}

1. Kerkhofs TM, Verhoeven RH, Van der Zwan JM, et al. Adrenocortical carcinoma: a population-based study on incidence and survival in the Netherlands since 1993. Eur J Cancer 2013;49: 2579-2586.
2. Gaujoux S, Brennan MF. Recommendation for standardized surgical management of primary adrenocortical carcinoma. Surgery 2012;152:123-132.

3. Kendrick ML, Lloyd R, Erickson L, et al. Adrenocortical carcinoma: surgical progress or status quo? Arch Surg 2001;136:543-549. 
4. Else T, Kim AC, Sabolch A, et al. Adrenocortical carcinoma. Endocr Rev 2014;35:282-326

5. Fassnacht M, Dekkers OM, Else T, et al. European Society of Endocrinology Clinical Practice Guidelines on the management of adrenocortical carcinoma in adults, in collaboration with the European Network for the Study of Adrenal Tumors. Eur J Endocrinol 2018;179:G1-46.

6. Polat $B$, Fassnacht $M$, Pfreundner $L$, et al. Radiotherapy in adrenocortica carcinoma. Cancer 2009;115:2816-2823.

7. Sabolch A, Feng M, Griffith K, et al. Adjuvant and definitive radiotherapy for adrenocortical carcinoma. Int J Radiat Oncol Biol Phys 2011;80:1477-1484.

8. Habra MA, Ejaz S, Feng L, et al. A retrospective cohort analysis of the efficacy of adjuvant radiotherapy after primary surgical resection in patients with adrenocortical carcinoma. J Clin Endocrinol Metab 2013;98:192-197.

9. Fassnacht M, Hahner S, Polat B, et al. Efficacy of adjuvant radiotherapy of the tumor bed on local recurrence of adrenocortical carcinoma. J Clin Endocrinol Metab 2006;91:4501-4504.

10. Sabolch A, Else T, Griffith KA, et al. Adjuvant radiation therapy improves local control after surgical resection in patients with localized adrenocortical carcinoma. Int J Radiat Oncol Biol Phys 2015;92:252-259.

11. Nelson DW, Chang SC, Bandera BC, et al. Adjuvant radiation is associated with improved survival for select patients with non-metastatic adrenocortical carcinoma. Ann Surg Oncol 2018;25:2060-2066

12. Gharzai LA, Green MD, Griffith KA, et al. Adjuvant radiation improves recurrence-free survival and overall survival in adrenocortical carcinoma. J Clin Endocrinol Metab 2019;104:3743-3750.

13. Shah MH, Goldner WS, Benson AB III, et al. NCCN Clinical Practice Guidelines in Oncology: Neuroendocrine and Adrenal Tumors. Version 2.2020. Accessed July 24, 2020. To view the most recent version, visit NCCN.org

14. Magee BJ, Gattamaneni HR, Pearson D. Adrenal cortical carcinoma: survival after radiotherapy. Clin Radiol 1987;38:587-588.

15. Markoe AM, Serber W, Micaily B, et al. Radiation therapy for adjunctive treatment of adrenal cortical carcinoma. Am J Clin Oncol 1991;14:170-174.
16. Pommier RF, Brennan MF. An eleven-year experience with adrenocortical carcinoma. Surgery 1992;112:963-970.

17. Hermsen IG, Groenen YE, Dercksen MW, et al. Response to radiation therapy in adrenocortical carcinoma. J Endocrinol Invest 2010;33: 712-714.

18. Viani GA, Viana BS. Adjuvant radiotherapy after surgical resection for adrenocortical carcinoma: a systematic review of observational studies and meta-analysis. J Cancer Res Ther 2019;15(Suppl): S20-26

19. Luo $Y$, Chen SS, Zheng XG, et al. The efficacy of radiation therapy in adrenocortical carcinoma: a propensity score analysis of a populationbased study. Medicine (Baltimore) 2017;96:e6741.

20. Darby S, McGale $\mathrm{P}$, Correa $\mathrm{C}$, et al. Effect of radiotherapy after breastconserving surgery on 10-year recurrence and 15-year breast cancer death: meta-analysis of individual patient data for 10,801 women in 17 randomised trials. Lancet 2011;378:1707-1716.

21. Folkesson J, Birgisson H, Pahlman L, et al. Swedish Rectal Cancer Trial: long lasting benefits from radiotherapy on survival and local recurrence rate. J Clin Oncol 2005;23:5644-5650.

22. Wadsley JC, Bentzen SM. Investigation of relationship between change in locoregional control and change in overall survival in randomized controlled trials of modified radiotherapy in headand-neck cancer. Int J Radiat Oncol Biol Phys 2004;60: 1405-1409.

23. Vanbrabant T, Fassnacht M, Assie G, et al. Influence of hormonal functional status on survival in adrenocortical carcinoma: systematic review and meta-analysis. Eur J Endocrinol 2018;179: 429-436.

24. Beuschlein F, Weigel J, Saeger W, et al. Major prognostic role of Ki67 in localized adrenocortical carcinoma after complete resection. J Clin Endocrinol Metab 2015:100:841-849.

25. Bilimoria KY, Shen WT, Elaraj D, et al. Adrenocortical carcinoma in the United States: treatment utilization and prognostic factors. Cancer 2008. 113:3130-3136.

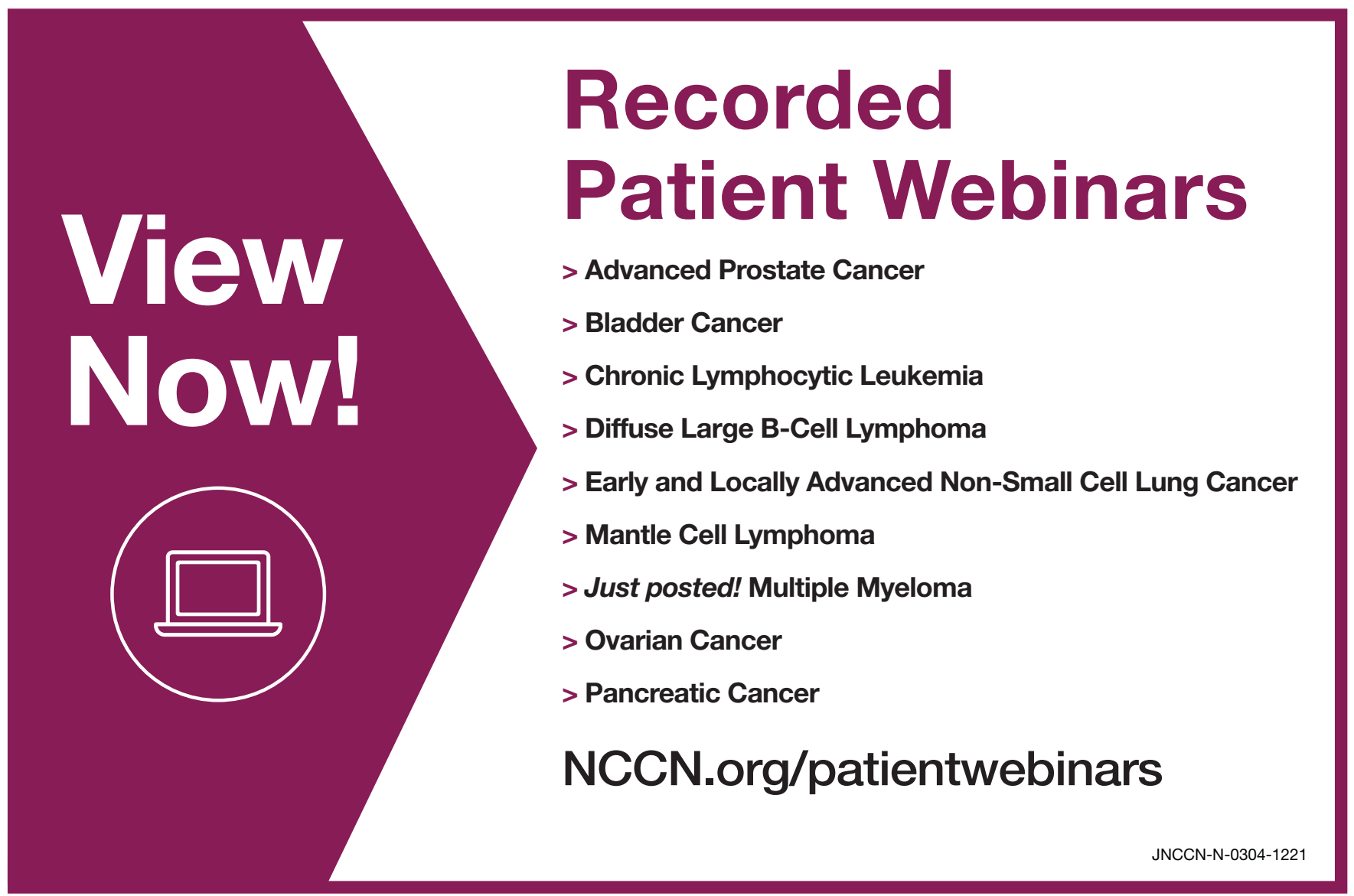

